\title{
A RELAÇÃO DA PREVISÃO DA PRECIPITAÇÃO PLUVIOMÉTRICA E CASOS DE DENGUE NOS ESTADOS DE ALAGOAS E PARAÍBA NORDESTE DO BRASIL.
}

\author{
EDIVANIA DE ARAUUJO LIMA; JANNE LÚCIA DA NÓBREGA FIRMINO; \\ MANOEL F. GOMES FILHO
}

\author{
Universidade Federal de Campina Grande - UFCG Av. Aprígio Veloso, 882 - Bodocongó - \\ CEP 58109-970- Campina Grande, Paraíba. \\ edy_al@hotmail.com.
}

Recebido Dezembro 2006 - Aceito Maio 2008

\begin{abstract}
RESUMO
Este trabalho apresenta um método para a previsão da distribuição espacial de novos casos de dengue nos estados de Alagoas e Paraíba (nordeste do Brasil), a partir da previsão da precipitação obtida por modelos atmosféricos regionais; esta estimativa é realizada a partir da redução de escala (downscaling) da previsão do modelo global ECHAM 4.5 para a escala do modelo regional RSM do NCEP, seguida de uma regressão matemática. Foram encontrados resultados satisfatórios para ambas as localidades, onde se observou uma boa relação entre a precipitação prevista e os casos de dengue observados e previstos. Verificou-se também, que a previsão da precipitação utilizando o modelo regional RSM aproximou-se bastante dos valores da precipitação observada nestas localidades, evidenciando que a previsão da distribuição espacial da dengue utilizando-se este método é bastante satisfatória.
\end{abstract}

Palavras Chaves: modelos de previsão, precipitação, downscaling, dengue;

\begin{abstract}
ON THE RELATION OF FORECAST OF PRECIPITATION AND THE CASES OF DENGUE IN THE STATES OF ALAGOAS AND PARAÍBA NORTHEAST BRAZIL

This work presents a method to estimate the spatial distribution of new cases of dengue disease in the States of Alagoas and Paraíba, Northeast Brazil. The estimative is obtained through downscaling the ECHAM 4.5 Global Model precipitation to the regional spatial scale of the NCEP's RSM model and then, performing a linear mathematical regression. The estimated and observed cases of dengue in both locals are in good agreement, evidencing a good relationship between the estimated precipitation and the actual cases of dengue. As one can see, the precipitation forecasted through these models represents a good approximation to the observed values, which has positively influenced the results presented here for the dengue disease.
\end{abstract}

Keywords: numerical prediction, precipitation, downscaling, dengue.

\section{INTRODUÇÃO}

O nordeste do Brasil se distingue do ponto de vista climático das demais regiões brasileiras não somente por suas secas recorrentes e pela baixa pluviometria média anual em sua porção semi-árida, mas principalmente pelo alto grau de previsibilidade da pluviometria média sazonal sobre a região, para a previsão da precipitação de curto prazo podem ser utilizados modelos numéricos do tempo que estimulam o estado da atmosfera por meio de modelos físico-matemáticos resolvidos numericamente através de técnicas computacionais avançadas (Nobre, et al., 2004).

Um dos ramos da meteorologia menos explorado até o presente momento no Brasil, é o que trata da influência dos parâmetros atmosférico-meteorológicos sobre o organismo dos seres vivos (biometeorologia), ainda assim, os poucos grupos que estão trabalhando nessa direção tem explorado muito mais essa influência sobre as plantas e os animais que sobre os seres humanos.

As causas da influência do tempo e do clima estão longe de serem totalmente conhecidos, logo é de grande importância à elaboração de estudos relacionando clima e saúde humana, pois esta é uma área de crescente preocupação por parte de médicos e climatologistas (Glasser, 1997).

Segundo informações do Projeto de Vigilância Epidemiológica (PROVEP), atualmente os sorotipos 1 e 2 da dengue distribuem-se por todo o Brasil e o sorotipo 3 foi observado desde 2002 ,no estado da Paraíba no qual o índice de infestação predial é em torno de $10 \%$. Já no estado de Alagoas cerca de 32 municípios apresentam médio risco em relação à 
doença ao passo que 10 municípios apresentam risco elevado com infestação acima de 3\% (FUNASA, 2004). No estágio atual do trabalho, envolvendo a dengue, têm-se clareza de sua estreita relação com os fatores climáticos, inclusive, correlações matemáticas significantes com os totais pluviométricos no mês de observação e antecedentes.

Os modelos preditivos para a transmissão de dengue têm enfatizado a importância da temperatura, precipitação e umidade relativa do ar sobre a distribuição do Aedes aegypti (mosquito transmissor) e na ocorrência de surtos de dengue sendo a precipitação o fator climático mais importante para esses modelos (Koopman et al., 1991; Hoop \& Foley, 2001).

Diante do que foi relatado sobre a influência dos parâmetros meteorológicos em relação à incidência da dengue no Brasil, em especial nos estados de Alagoas e Paraíba, o objetivo deste é estabelecer um sistema que permite prever a distribuição espacial de casos de dengue nos estado de Alagoas e Paraíba, nos quais, a previsão da precipitação sazonal é realizada com sucesso pelos modelos atmosféricos. Tal sistema de previsão será possível a partir da redução de escala (downscaling) da previsão de precipitação do modelo global para a escala regional, que inclui a região em estudo, sendo realizada posteriormente a regressão múltipla, utilizando as condições térmicas do oceano Atlântico, índice de Oscilação Sul, números de casos de dengue notificados nestas localidades e a precipitação prevista pelo modelo e observada nos dois estados durante o período de janeiro de 2000 a dezembro de 2002.

\section{DADOS E METODOLOGIA}

Os dados utilizados neste trabalho foram: totais mensais de precipitação registrados nos postos pluviométricos dos estados de Alagoas e Paraíba, adquiridos através da Diretoria de
Hidrometeorologia de Alagoas - DHM/AL e Instituto Nacional de Meteorologia - INMET $3^{\circ}$ Distrito, localizado na cidade de Recife - PE; precipitação obtida através do modelo RSM; totais mensais de casos notificados de dengue nos dois estados, obtidos através da Secretária Estadual de Saúde no setor de vigilância epidemiológica do SUS (Sistema Único de Saúde); valores mensais do Índice de Oscilação Sul (IOS), Anomalias de Temperatura da Superfície do Mar (TSM) do Atlântico Norte e Sul, extraídos da Climate Diagnostics Bulletin Near Real - Time analyses Ocean/Atmosphere durante o período de 2000 a 2002.

Para a obtenção da previsão de precipitação para os estados de Alagoas e Paraíba, foram utilizadas as saídas do modelo global ECHAM 4.5, da Universidade de Hamburgo, disponibilizado através do Centro Europeu de Previsão do Tempo de Médio Prazo (ECMWF), as saídas desse modelo global foram utilizadas para inicializar o modelo regional RSM do NCEP dos Estados Unidos, essa metodologia de previsão vem sendo adotada operacionalmente na Fundação Cearense de Meteorologia e Recursos Hídricos (FUNCEME).

O RSM97 foi integrado com um conjunto de 10 membros na versão não-hidrostática, intervalo de tempo de 600 segundos em modo de simulação, com um espaçamento de grade de 60 $\mathrm{km}$ centrada na latitude de $30 \mathrm{o}$ Sul, na longitude de $27^{\circ}$ Leste, e 18 níveis verticais. $\mathrm{O}$ aninhamento entre o RSM97 e o ECHAM 4.5 foi executado de forma unidirecional, com condições iniciais distintas em cada um dos 10 membros, ou seja, a forçante de grande escala, vento, pressão, temperatura e umidade específica do ar, são inicializados pelo ECHAM 4.5 e repassados ao RSM97 em todo o domínio, e não somente nas fronteiras laterais, a cada 6 horas, em cada um dos 10 membros do conjunto nas integrações de forma distinta, maiores esclarecimentos encontram-se descritos em Silva \& Castro (2005).

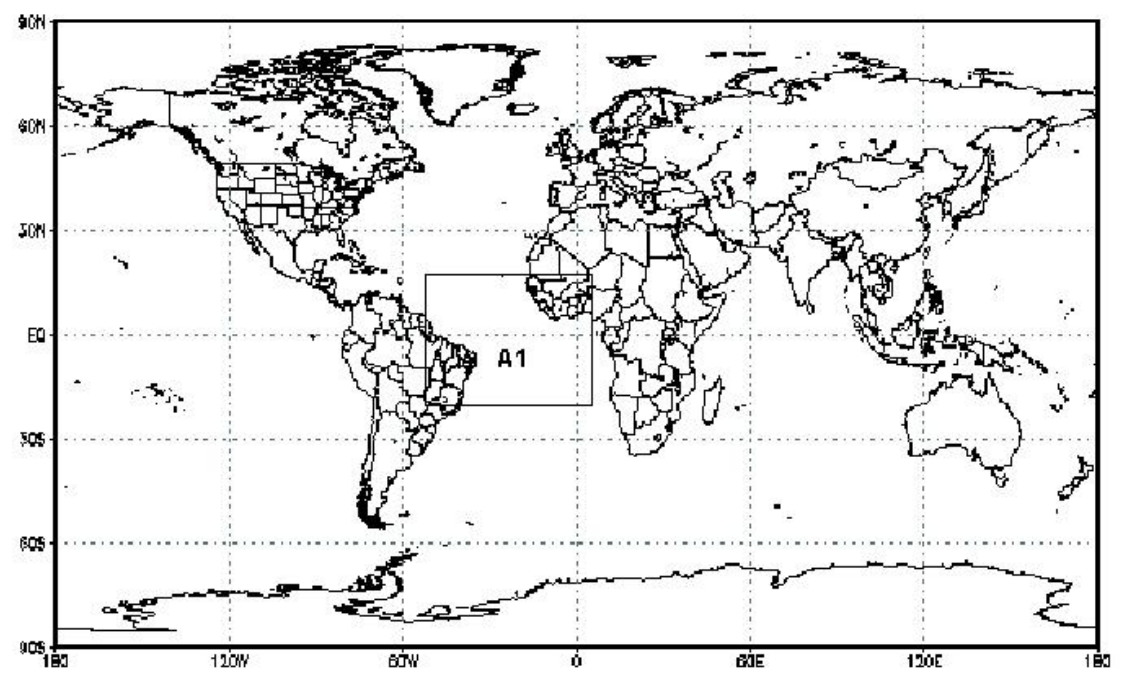

Figura 1 - Domínio do Modelo Global ECHAM 4.5 utilizado como forçante de entrada nas simulações do RSM97. Fonte: Silva \&Castro, 2005. 

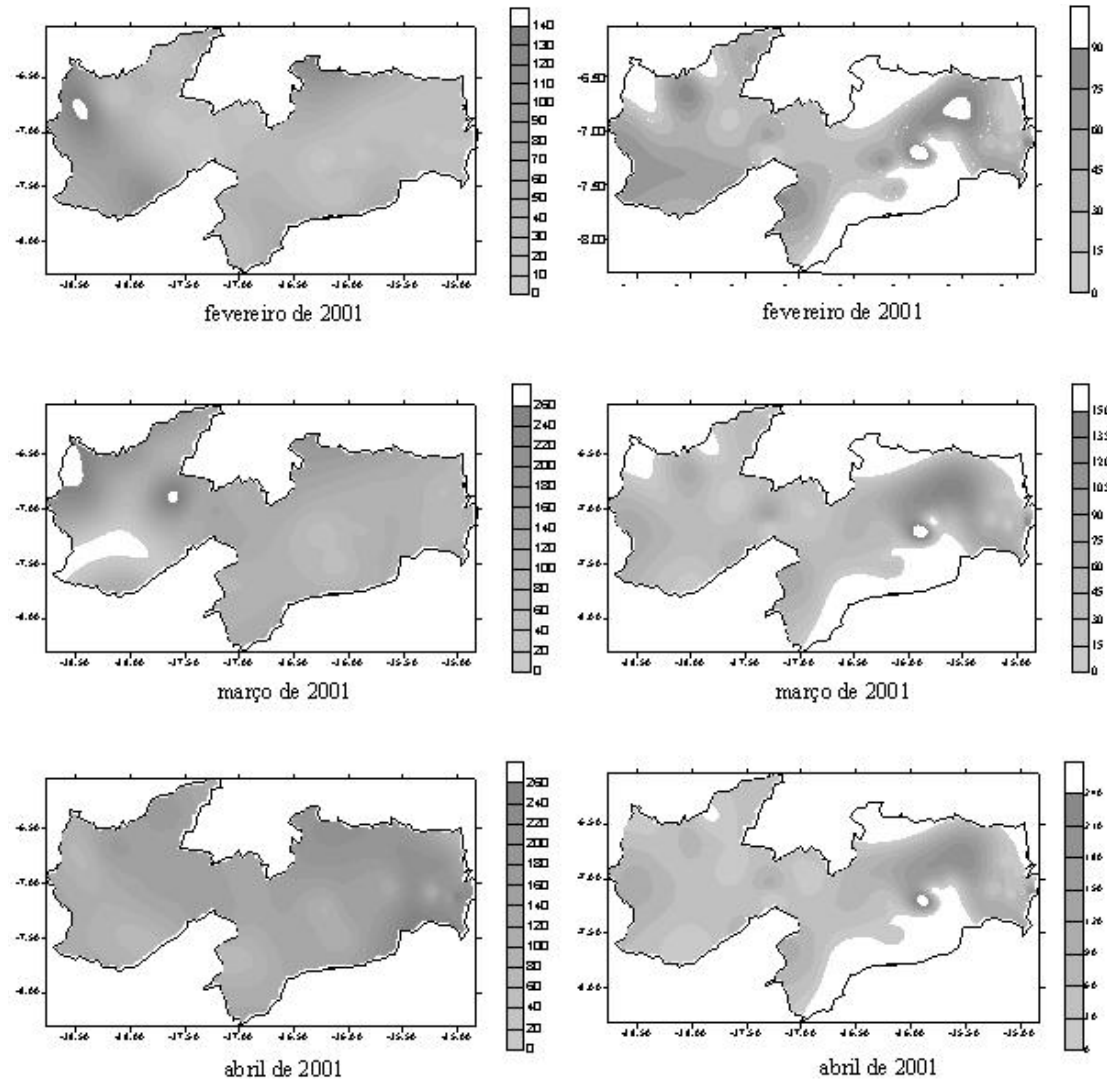

Figura 2 - Distribuição espacial da precipitação prevista (esquerda) e do número de casos do dengue previsto (direita) para a Paraíba na estação chuvosa do ano 2000, meses de fevereiro a abril.

Posteriormente, utilizando o Stat Soft'99 Edition foi realizada a regressão linear múltipla Spiegel (1985), através do método de mínimos quadrados (buscando encontrar a reta que melhor se ajusta aos pontos). Na regressão utilizaramse como variáveis independentes a precipitação prevista no modelo, o índice de oscilação sul e anomalias de temperatura da superfície do mar do Atlântico norte e sul, e como variável dependente foi utilizada os casos notificados de dengue

$$
y_{i}=e_{1}+b_{0} x_{0}+b_{1} x_{1 i}+b_{2} x_{2 i}+b_{3} x_{3 i}+b_{4} x_{4 i} \ldots b_{n} x_{n i}
$$

onde $\mathrm{Y}_{\mathrm{i}}$ é a variável dependente (casos de dengue previsto); $X_{i}$ são as variáveis independentes (precipitação prevista, IOS, anomalias do Atlântico norte e sul); $b_{i}$ são as contribuições de cada variável independente e $\mathrm{e}_{\mathrm{i}}$ é o erro aleatório do modelo.

O objetivo da técnica de regressão linear múltipla foi determinar as relações entre a variável dependente (dengue) e o conjunto de variáveis independentes, ou seja, a regressão foi utilizada para testar dependências cumulativas de uma única variável dependente em relação a diversas variáveis independentes. Cada variável foi isolada e mantida constante enquanto as variáveis restantes variaram sistematicamente, sendo observados os seus efeitos sobre a variável dependente.

Os Totais mensais de precipitação registrados nos postos pluviométricos dos dois estados serviram para que verificássemos a coerência ente o valor previsto e os observados da precipitação, ao mesmo tempo buscou-se observar a relação da precipitação observada com os casos de dengue notificados nas duas localidades.

Foram utilizados os aplicativos gráficos SURFER 7.0, na confecção de mapas e o GrADS (Grid Analysis and Display System), sendo este último utilizado para a obtenção dos valores numéricos da precipitação prevista pelo modelo.

A partir dos resultados da regressão linear múltipla, foi realizada a previsão de casos de dengue. Daí, usando as variáveis atmosféricas, foi realizado o cálculo dos desvios médios das variáveis (dengue prevista e casos de dengue notificados) possibilitando assim observar a incerteza do modelo. A incerteza da previsão foi calculada com sendo a diferença entre o valor do desvio médio experimental e o desvio médio real dos casos de dengue.

$$
\mathrm{D}_{\text {Previsto }}-\mathrm{D}_{\text {Observado }}=\text { Erro }
$$




\section{RESULTADOS E DISCUSSÃO}

Nas Figuras 2 e 3 observou-se uma relação bem definida entre a precipitação prevista e os casos de dengue observados nos estados de Alagoas e Paraíba, entretanto essa relação parece estar defasada por um período de um a dois meses. Percebeu-se ainda, uma variabilidade espacial considerável na distribuição dos casos de dengue entre as regiões do agreste, litoral e sertão no território paraibano (Figura 2) e nas regiões do agreste e litoral alagoano (Figura 3), o que pode ser explicado pelos diferentes sistemas que provocam a precipitação nestas localidades.

No geral, verificou-se que as epidemias da doença iniciam-se na estação menos úmida, sugerindo uma circulação viral estreitamente relacionada com o início da estação mais quente do ano, este resultado também foi observado em Sousa (1999), quando autor relata que mesmo em períodos com menor pluviosidade o mosquito transmissor da doença Aedes aegypti pode reproduzir-se dentro das residências, ou seja, os mosquitos

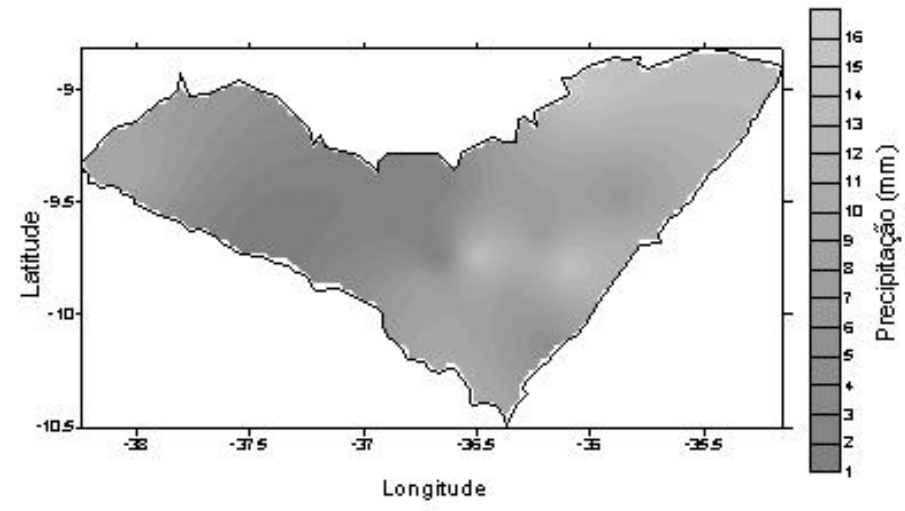

abril de 2001

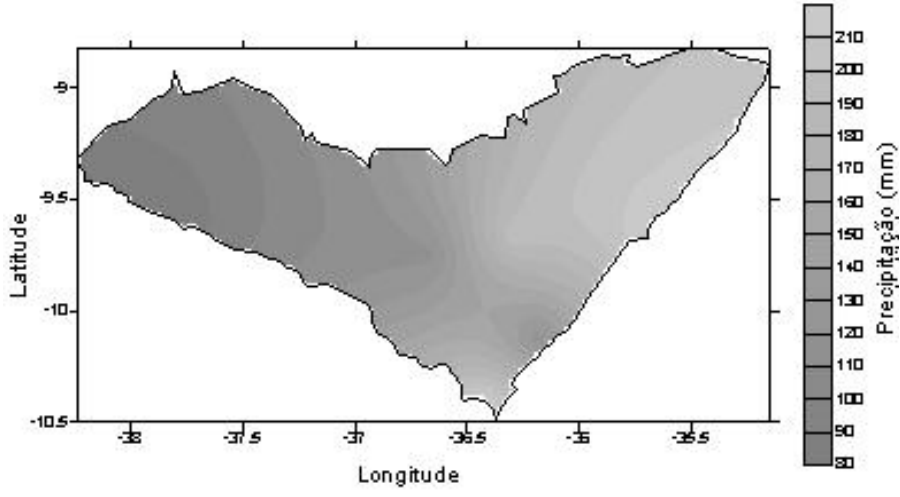

maio de 2001 depositam seus ovos durante o período mais chuvoso e os mesmos eclodem quando os totais pluviométricos diminuem e as temperaturas se elevam.

Na Figura 2, representante do estado da Paraíba pode-se observar claramente nos meses de fevereiro e março a afirmação descrita acima. Entretanto no mês de abril de 2001, os máximos valores de dengue surgiram exatamente nas localidades com maiores totais pluviométricos.

O estado de Alagoas (Figura 3) apresentou resultados semelhantes aos observados para o estado da Paraíba, ou seja, nas regiões onde foram previstos menores totais pluviométricos também foram observados os maiores indícios de ocorrência para a enfermidade. Na literatura trabalhos como de Consoli \& Oliveira (1994), Glasser (1997), Glasser e Gomes (2002), entre outros afirmam que a temperatura tem influência direta na distribuição geográfica de populações vetores de doenças e o acúmulo de água em reservatórios durante os períodos sem chuvas, associado à ocorrência de temperaturas elevadas, torna favorável a reprodução do vetor transmissor.

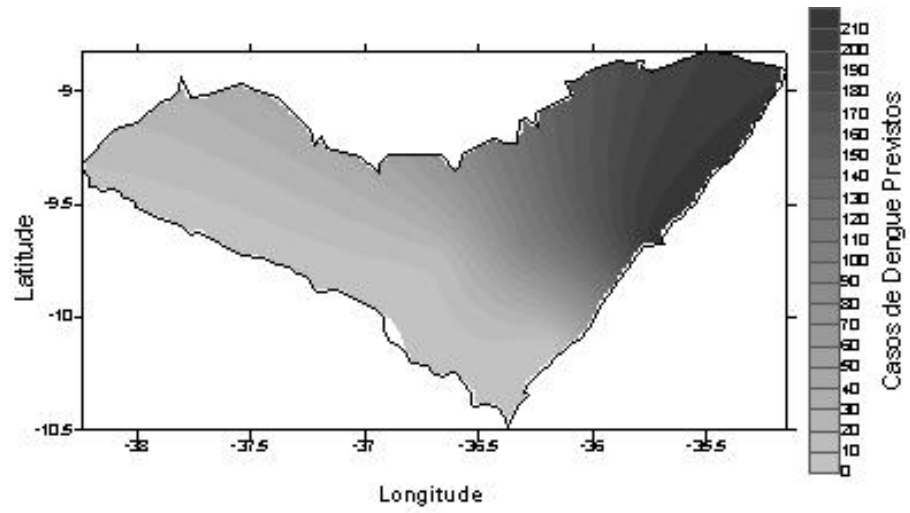

abril de 2001

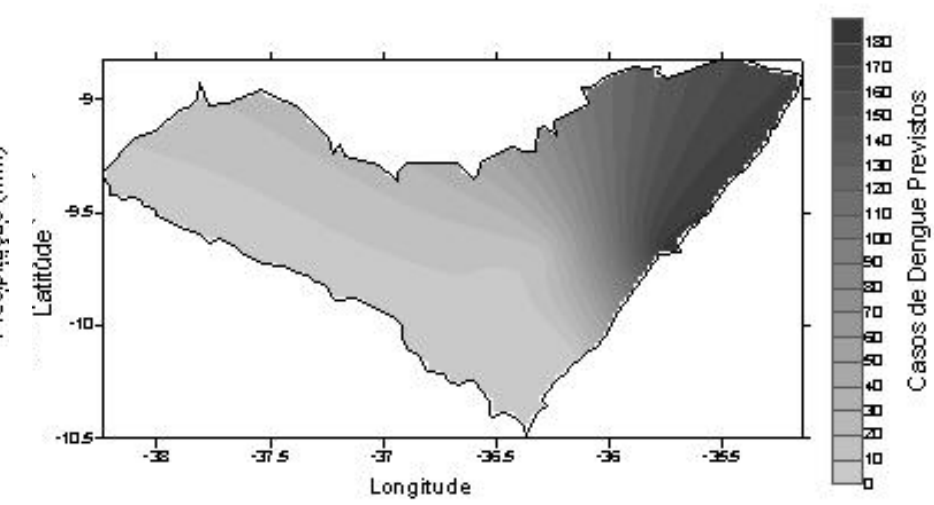

maio de 2001

Figura 3 - Distribuição espacial da precipitação prevista (esquerda) e do número de casos do dengue previsto (direita) para Alagoas na estação chuvosa do ano 2000, meses de abril e maio. 
As figuras 4 e 5 apresentam comparações entre os valores de dengue previsto pelo modelo de regressão e os valores de casos de dengue notificados para duas cidades do estado da Paraíba: Cajazeiras, cidade representativa do sertão paraibano (6 $6^{\circ} 53^{\prime} 23^{\prime}$ 'S; 38 33'36"W) e João Pessoa (709'28"; $\left.34^{\circ} 47^{\prime} 30^{\prime \prime} \mathrm{W}\right)$, localizada na faixa leste do estado. Observa-se nessas figuras que houve uma super estimação nos valores de dengue previsto em relação ao dengue observado na cidade de Cajazeiras (Figura 4), o mesmo ocorrendo com a cidade de João Pessoa (litoral paraibano) (Figura 5) nos meses em que os casos de dengue observados apresentaram um pico, evidenciando que há uma tendência do modelo RSM de sempre subestimarem as variáveis previstas, quando existem picos nos dados observados dessas variáveis, ou seja, o modelo não se ajusta a picos.

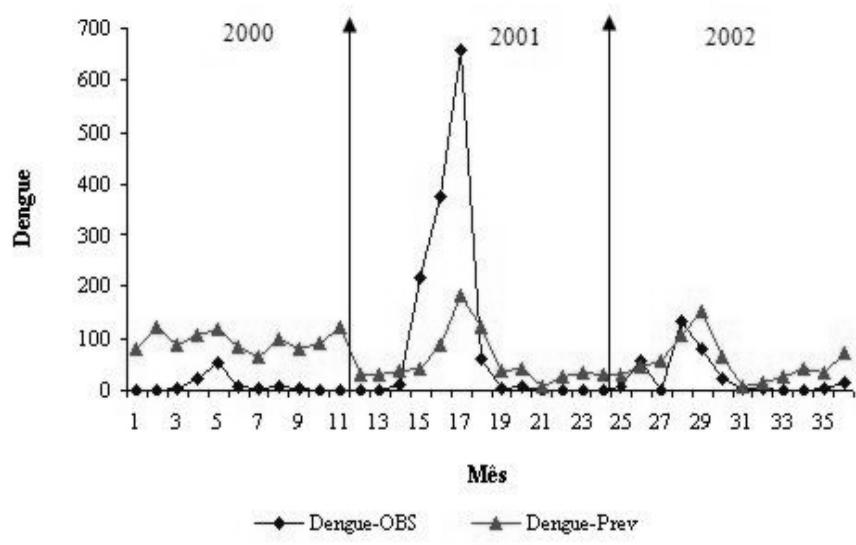

Figura 4 - Diagrama comparativo do dengue previsto (Dengue-Prev) pela regressão linear múltipla, com os casos de dengue observado (Dengue-OBS) para a cidade de Cajazeiras (6 53'23“'S; 38 33'36”'W) no período de 2000 a 2002.

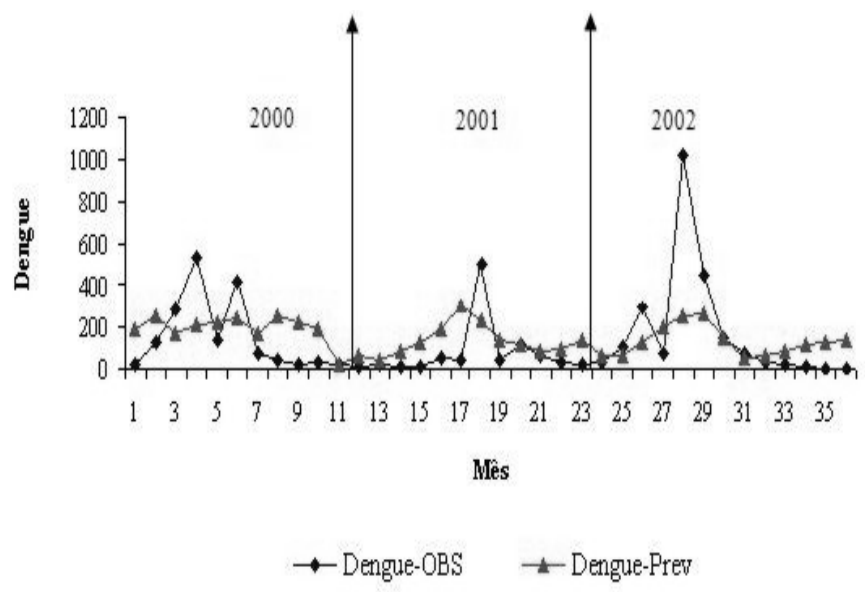

Figura 5 - Diagrama comparativo do dengue previsto (Dengue-Prev) pela regressão linear múltipla, com os casos de dengue observado (Dengue-OBS) para a cidade de João Pessoa no período de 2000 a 2002 .

\section{CONCLUSÃO}

Considerando os resultados obtidos, pode-se concluir que:

- a análise da distribuição espacial da precipitação mensal e dos casos de dengue nos estados de Alagoas e Paraíba permitiu observar a relação direta entre essa variável meteorológica e a doença que é transmitida por vetores e que está intrinsecamente ligada às variações climáticas.

- analisando a variação sazonal de dengue, observouse que durante períodos mais úmidos, a distribuição espacial da doença apresentou-se menos intensa, enquanto que nos períodos mais secos as possibilidades de ocorrência aumentam, confirmando a sazonalidade da doença.

- através da regressão linear múltipla, que utilizou a precipitação prevista pelo modelo RSM, o índice de oscilação sul e as anomalias de temperatura da superfície do mar, foi possível prever a distribuição espacial dos casos de dengue nos dois estados, entretanto devido às limitações do método, em alguns casos, o modelo regressivo não foi capaz de reproduzir os resultados esperados. Sugere-se que em uma etapa futura, seja feito uso de um modelo não-linear, para melhorar a previsão, bem como a utilização de funções ortogonais empíricas, para melhor ajuste da probabilidade.

- a previsão da distribuição espacial da dengue utilizando-se a previsão pluviométrica a partir de modelos regionais é válida, uma vez que a distribuição espacial da precipitação influencia diretamente na distribuição e intensidade de ocorrências da doença.

Espera-se que este trabalho possa contribuir para um melhor controle desta enfermidade, uma vez que será possível prever com certa antecedência quais localidades poderão sofrer epidemias da doença, facilitando assim um melhor controle epidemiológico por parte das autoridades competentes.

\section{REFERÊNCIAS BIBLIOGRÁFICAS}

CONSOLI, R. A. G. B.; OLIVEIRA, L. Principais mosquitos de importância sanitária no Brasil. 1ed. Rio de Janeiro: Fiocruz, 1994.228 p.

FUNASA. Situação epidemiológica de dengue no Brasil (20012002). <http://www.saude.pr.gov.br/Seminario/Dengue/ funasa.htm> . 14 de janeiro de 2004.

FUNCEME. Projeto Downscaling. <http://www.funceme. br/demet/ds/equipe.htm>. 9 de março de 2004.

GLASSER, C. M. Estudo da Infecção do estado de São Paulo por Aedes aegypti e Aedes albopictus. São Paulo. 1997. 93f. Dissertação (Mestrado em Saúde Pública) - Faculdade de Saúde Pública da USP.

GLASSER, C.M.; GOMES, A. C. Clima e Sobreposição da distribuição de Aedes aegypti e Aedes albopictus na 
infestação do estado de São Paulo. Revista de Saúde Pública. São Paulo, v. 36, n. 2, p. 1666-1672, dez. 2002.

HOOP, M.; FOLEY, J. A. Global scale relationship between Climate and Dengue fever vector Aedes Aegypti. Climate Change, v. 48, n. 2-3, p. 441-463, fev 2001.

NOBRE, P; GALVÃO, C. O; GOMES FILHO, M. F. Previsibilidade climática sobre o Nordeste e os recursos hídricos. Campina Grande, 6p. Submetido a Revista Brasileira de Recursos Hídricos (RBRH), 2004.

KOOPMAN, J. S; PREVOTS, D. R.;VACA, M.; DANTES, H.G.;ZARATE, M. L. A.;LONGINI, Jr.; SEPULVEDA, A. Determinants and predictors of Dengue infection in México. Am Journal Epid. EUA, v. 133, n. 36, p. 1168-1178, set. 1991.
SILVA, E. M.; CASTRO, M. A. H. Análise da climatologia de precipitação simulada em escala regional usando downscaling dinâmico sobre a região norte do nordeste do Brasil . Revista Tecnológica. Fortaleza, v. 26, n.2, p. 124133, dez. 2005.

SPIEGEL, M. R. Estatística. $2^{\circ}$ ed. São Paulo: Schaum McGrawHill, 1985. $454 \mathrm{p}$.

SOUZA, S. R. Fatores associados á ocorrência de formas imaturas de Aedes aegypti na Ilha do Governador, Revista da Sociedade Brasileira de Medicina Tropical, , Rio de Janeiro, v.32, n.4, p.373-382, jul/ago, 1999. 